\title{
C
}

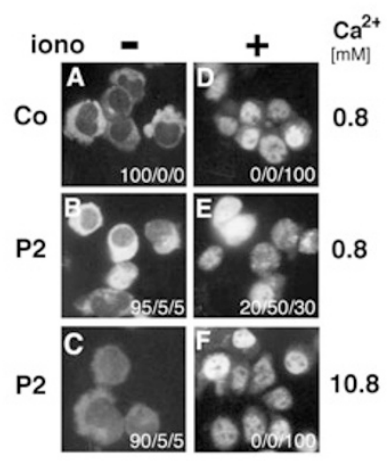

Figure 3 The calcium entry defect affects SCID patients' B cells and fibroblasts. (c) EBV-transformed B cell lines from patient 2 and a control were left unstimulated or stimulated with ionomycin in the presence of the indicated $\mathrm{CaCl} 2$ concentrations. Numbers in the panels refer to the approximate percentages of cells with cytoplasmic, partially nuclear and fully nuclear NFAT staining, respectively.

a

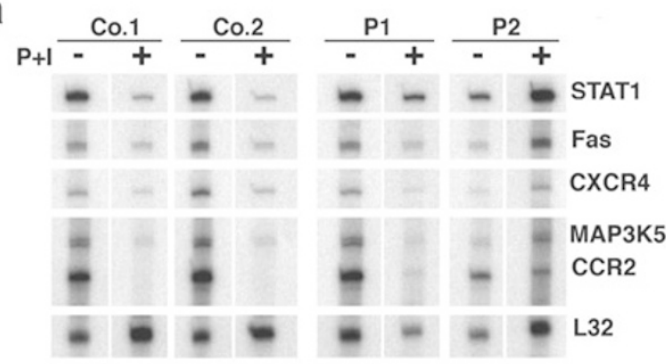

d

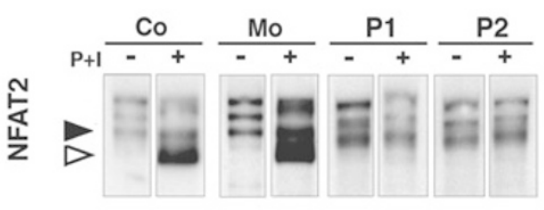

Figure $5 \mathrm{~T}$ cells deficient in calcium influx fail to repress the expression of certain genes. (a) RNase protection assays confirm down-regulation of five representative calcium-repressed genes. T cell lines from patients (P1, P2) and controls (Co1, Co2) were left unstimulated (-) or stimulated (+) with PMA + ionomycin and cytokine concentrations were assessed by RPA. (d) Lack of induction of the CsA-sensitive NFAT2 splice variant50 in the patients' T cells after prolonged stimulation. To confirm DNA array data showing that NFAT2 is induced in control T cells but not in patient T cells (see Fig. 6), T cells were stimulated with PMA + ionomycin $(\mathrm{P}+\mathrm{I})$ and NFAT2 was detected by immunoblotting using 7A6 mAb. Filled and open arrowheads indicate the phosphorylated and unphosphorylated forms of the short inducible NFAT2 isoform, respectively.

\section{Corrigendum: Connecting immunology research to public health: Cuban biotechnology}

\section{Agustin Lage}

Nat. Immunol. 9, 109-112 (2008); published online 18 January 2008; corrected after print 6 February 2008

In the version of this article initially published, the URL for the 2006 product survey of the Pharmaceutical Manufacturers Association is incorrect. The correct URL should be http://www.phrma.org/files/Biotech\%202006.pdf. 